\title{
Synthesis of highly integrated optical network based on microdisk-resonator add-drop filters in silicon-on-insulator technology
}

\author{
Andrzej Kaźmierczak \\ Fabian Dortu \\ Domenico Giannone \\ Multitel \\ Applied Photonics Department \\ 2 rue Pierre et Marie Curie \\ B-7000 Mons \\ Belgium
}

Wim Bogaerts

Ghent University

Interuniversity Microelectronics Center

Department of Information Technology

Sint-Pietersnieuwstraat 41

B-9000 Gent

Belgium

\author{
Emmanuel Drouard \\ Pedro Rojo-Romeo \\ Frederic Gaffiot \\ Université de Lyon \\ Institut des Nanotechnologies de Lyon \\ INL-UMR5270, CNRS \\ Ecole Centrale de Lyon \\ 36 avenue Guy de Collongue \\ F-69134 Ecully \\ France
}

\begin{abstract}
We analyze a highly compact optical add-drop filter topology based on a pair of microdisk resonators and a bus waveguide intersection. The filter is further assessed on an integrated optical $4 \times 4$ network for optical on-chip communication. The proposed network structure, as compact as $50 \times 50 \mu \mathrm{m}$, is fabricated in a CMOS-compatible process on a silicon-on-insulator (SOI) substrate. Finally, the experimental results demonstrate the proper operation of the fabricated devices. () 2009 Society of Photo-Optical Instrumentation Engineers. [DOI: 10.1117/1.3242841]
\end{abstract}

Subject terms: resonators; optical interconnects; optical circuits; silicon; integrated optics; waveguides.

Paper 090477R received Jun. 30, 2009; revised manuscript received Aug. 7, 2009; accepted for publication Aug. 11, 2009; published online Oct. 7, 2009.

\section{Introduction}

The whispering gallery mode concept has found a series of applications in the domain of integrated optics. In particular, numerous research groups all over the world have analyzed applications of microring and microdisk resonators, including wavelength selective optical filters, ${ }^{1}$ optical sensors, ${ }^{2}$ light modulators, ${ }^{3}$ and compact laser sources. ${ }^{4}$ For example, microring resonators enable building ultracompact and efficient wavelength switching devices. Ring resonators of radii as small as $1.5 \mu \mathrm{m}$ have been demonstrated in silicon-on-insulator (SOI) CMOS-compatible technology. ${ }^{5}$ Microdisks are beneficial with respect to microrings due to the lack of internal sidewalls, resulting in lower losses and generally higher quality factors. The main drawback of microdisks in comparison to microrings is the presence of first-order radial modes, which occupy the free spectral range (FSR) of the filter. This paper demonstrates microdisks as an interesting alternative solution to microrings for application in optical on-chip interconnection networks.

The standard topology of a photonic wavelength selective switch, or add-drop filter, based on a microdisk reso-

$\overline{0091-3286 / 2009 / \$ 25.00 \text { @ } 2009 \text { SPIE }}$ nator is composed of a pair of parallel bus waveguides coupled by a microdisk. ${ }^{1}$ This combination makes it possible to construct an add-drop filter almost as compact as the corresponding resonator. Unfortunately, for some more complex applications (e.g., optical networks) this configuration is not very favorable due to the reversed direction of the dropped signal with respect to the transmitted signal. ${ }^{6}$

\section{Optical Add-Drop Filter Design}

A modified add-drop filter topology, based on a waveguide intersection and a pair of microdisk resonators, makes it possible to propagate both the dropped and the transmitted signal in the same direction [Fig. 1(a)].

The topology based on a pair of microdisks instead of a single microdisk has central symmetry with respect to the waveguide intersection. This is especially beneficial when the waveguide crossings are lossy as it makes it possible to avoid passing the crossing twice when the signal originates from the opposite side of the crossing with respect to the single ring configuration, as shown in Fig. 2.

The symmetric configuration was already analyzed in a previous publication, ${ }^{7}$ where we demonstrated an add-drop filter based on resonators with disk radii as small as $2 \mu \mathrm{m}$. From these results, we further developed this concept and 


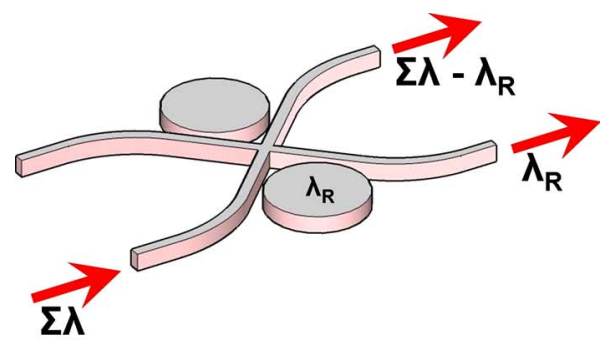

(a)

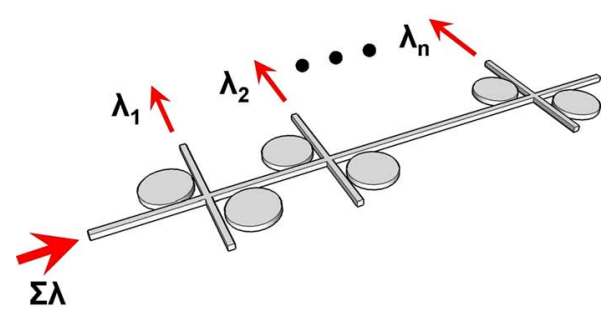

(b)

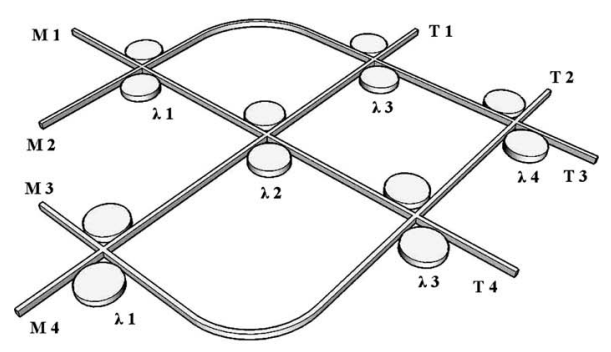

(c)

Fig. 1 (a) Optical add-drop filter based on a pair of microdisk resonators coupled by a waveguide intersection, (b) photonic $1 \rightarrow n$ network based on serially connected filters, and (c), schematic of a $4 \times 4$ integrated optical network.

studied the suitability of the topology for the construction of a compact, optical bidirectional network routed with wavelength-division multiplexing (WDM).

\section{Optical Network Principles}

The simplest network that can be assembled using the proposed filter topology is a $1 \rightarrow n$ network, where the filters are serially connected ${ }^{8}[$ Fig. 1(b)]. The network can be expanded into a multiple-input device when each filter has its ports connected to the ports of the other filters. A specific topology of such a fully bidirectional $n \times n$ network is shown schematically in Fig. 1(c) for $n=4$, as proposed by O'Connor and Gaffiot.

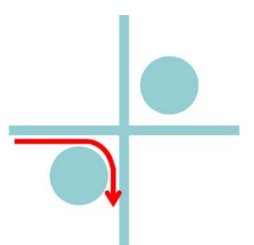

(a)

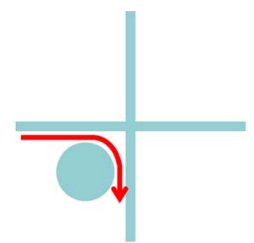

(b)

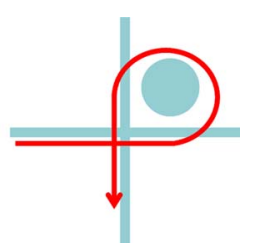

(c)
Fig. 2 Comparison of dropped signal route in the case of (a) a symmetric filter and (b) a nonsymmetric filter with the resonator in front of the crossing and (c) behind the crossing.
Table 1 The truth table of the optical $4 \times 4$ network.

\begin{tabular}{ccccc}
\hline \hline & T1 & T2 & T3 & T4 \\
\hline M1 & $\lambda 2$ & $\lambda 3$ & $\lambda 1$ & $\begin{array}{c}\text { Non resonant } \\
(\lambda 4)\end{array}$ \\
M2 & $\lambda 3$ & $\lambda 4$ & Non resonant & $\lambda 1$ \\
M3 & $\lambda 1$ & Non resonant & & \\
& & $(\lambda 2)$ & $\lambda 4$ & $\lambda 3$ \\
M4 & $\begin{array}{c}\text { Non resonant } \\
(\lambda 4)\end{array}$ & $\lambda 1$ & & $\lambda 3$ \\
\hline \hline
\end{tabular}

The network has a simple layout consisting of four bus waveguides, two straight and two 90-deg bents, composed appropriately to form six intersections in four rows (each row corresponding to a specific wavelength). These intersections form the center of six wavelength-selective filters.

The network can be seen as a model of a backbone structure for optical on-chip interconnects when the major functional blocks (e.g., processor cores, memory blocks, functional units, etc.) of an IC are connected to its in- and output ports (M1...M4, T1...T4). As the network is completely passive, optical nonblocking and bidirectional links are achieved when functional blocks are equipped with an optoelectronic transceiver circuit.

Although in the present example, a $4 \times 4$ optical network is shown, it can be expanded to larger structures, e.g., $12 \times 12$ or $16 \times 16$, by the addition of more add-drop filters rows. The multichannel operation of the network is detailed in Table 1.

Because of the diagonal nonresonant routing [Fig. 1(a)], the communication can be established between each input and output port of the network. Furthermore, a set of four wavelengths matching the resonant wavelengths of the adddrop filters is sufficient to provide communication within the network. Indeed, the filters of the first $(\lambda 1)$ and third $(\lambda 3)$ rows act as wavelength-selective switches regardless of the input port, while one of the other filters $(\lambda 2$ or $\lambda 4)$ is either bypassed or, to be passed through, light must be dropped at the preceding filter (which obviously has a different resonant wavelength). The operation table can be expanded accordingly with the expansion of the optical network.

\section{Choice of Add-Drop Filter Parameters for Optical Network Design}

To examine the suitability of the symmetric add-drop filter for the construction of an optical network, we designed and implemented a $4 \times 4$ demonstration structure. A set of four parameters influence the wavelength response of the adddrop filter (Fig. 3), namely, the bus waveguide height $(h)$ and width $(w)$, the resonator radius $(R)$, and the coupling gap between the bus waveguide and the resonator $(d)$.

In this study, the resonant wavelength of the filters are adapted by varying the resonator radii $(1.8,1.9,2.0$, and $2.1 \mu \mathrm{m})$, while keeping the other parameters fixed. This strategy has the advantage of making the fabrication pro- 


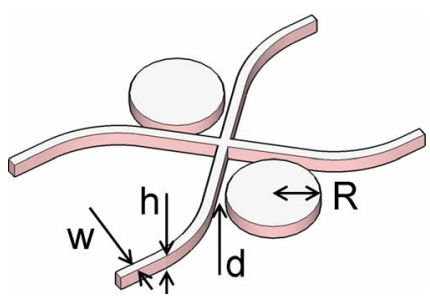

Fig. 3 Four geometrical parameters of the add-drop filter.

cess easier. The other filter parameters were adapted to match the Interuniversity microelectronics center (IMEC) fabrication technology for photonic wires on a SOI substrate, ${ }^{10}$ i.e., $d=200 \mathrm{~nm}, h=220 \mathrm{~nm}$, and $w=350 \mathrm{~nm}$.

The transmitted and dropped signal spectra in the wavelength range 1450 to $1600 \mathrm{~nm}$ were simulated with the 3-D finite-difference time-domain (FDTD) software (Fig. 4). Three groups of resonant peaks and dips are present around 1470,1520 , and $1580 \mathrm{~nm}$. One can notice that the group of peaks located around $1580 \mathrm{~nm}$ offers the best dropping efficiency, while being separated one from another by only about $3 \mathrm{~nm}$. The best resonant wavelength separation occurs in the proximity of $1470 \mathrm{~nm}$, where the peaks are separated by about $10 \mathrm{~nm}$. The group of peaks located around $1520 \mathrm{~nm}$ (close-up of Fig. 4) can be seen as a sort of compromise between peak separation and dropping efficiency. There are four resonance peaks present at the central wavelengths of $1507.34,1513.97,1520.18$, and $1525.96 \mathrm{~nm}$, corresponding to microdisks of radii 1.8, 1.9, 2.0 , and $2.1 \mu \mathrm{m}$, respectively. The quality factor of these peaks varies from 1500 to 2100 and dropping efficiency varies from 0.48 to 0.80 . In the proximity of $1.535 \mu \mathrm{m}$ one

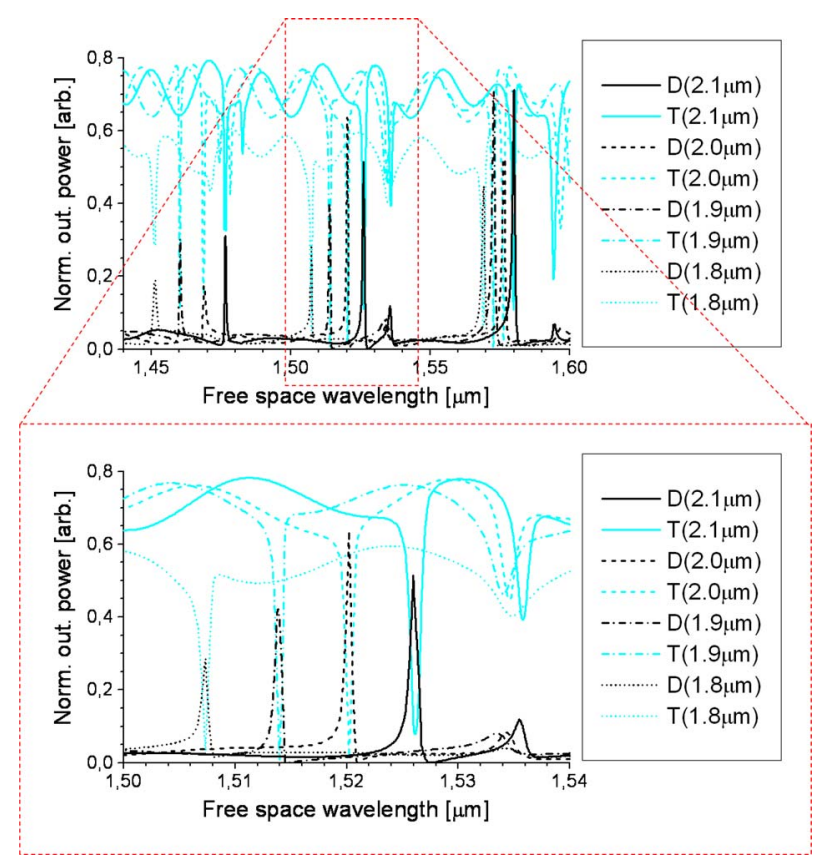

Fig. 4 Three-dimensional FDTD simulation of transmission spectra of add-drop filters with microdisk resonator radii ranging from 1.8 to $2.1 \mu \mathrm{m}$ (D refers to the dropped signal and $T$ refers to the nonresonant transmitted signal).

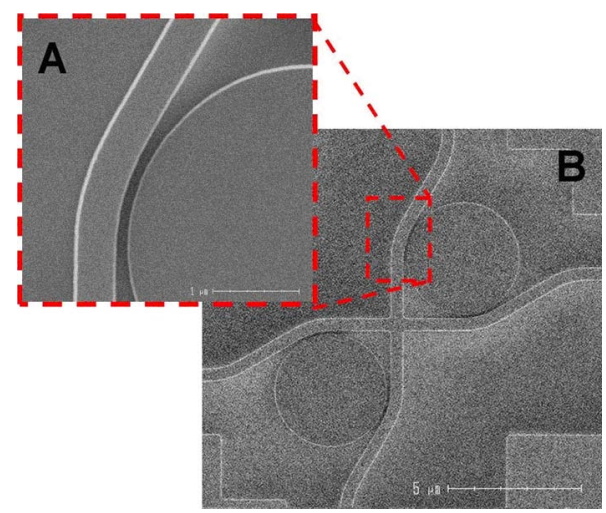

Fig. 5 Scanning electron microscopy (SEM) images of the add-drop filter: (a) close up of a coupling section and (b) general view of the structure.

can observe a secondary peaks corresponding to the first radial order resonances. Note that by pure coincidence, for this particular wavelength range, the secondary peaks are located in very near proximity.

\section{Fabrication and Experimental Validation of Add-Drop Filters}

The devices were fabricated at IMEC following the aforementioned waveguide and ring dimensions. However, as the simulation showed a relatively low dropped signal level, the design was slightly modified to enhance coupling efficiency between the bus waveguides and the microdisk resonators. To do so without deteriorating the transmission properties of the bus waveguides, we introduced an angled coupling waveguide sections, wrapping around the resonator (Fig. 5).
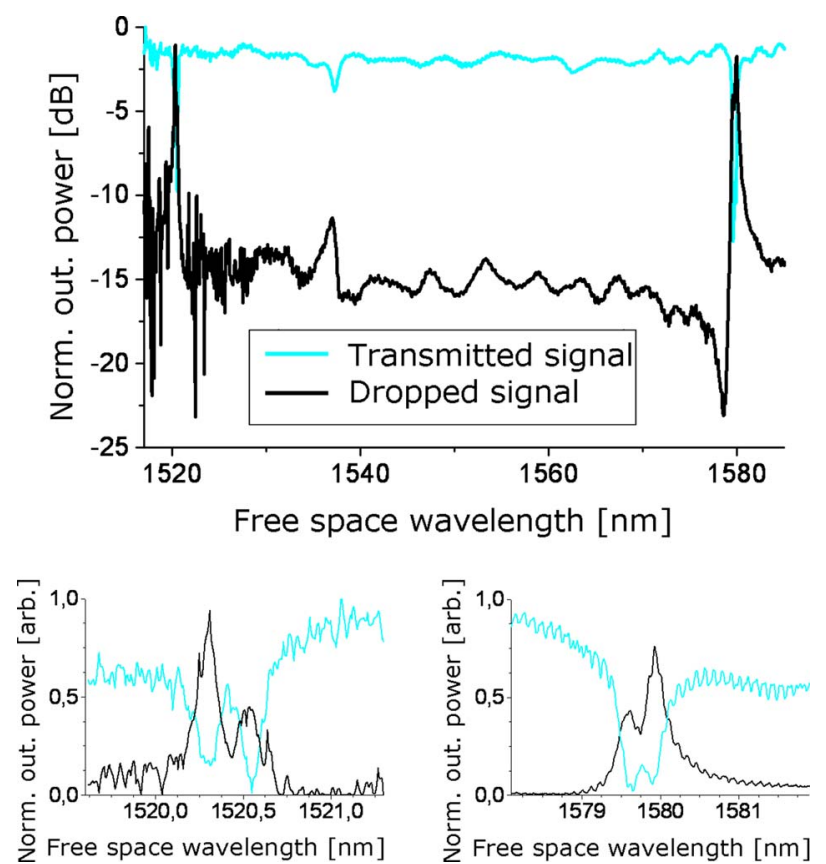

Fig. 6 Experimental transmitted (cyan) and dropped (black) spectra of the optical add drop filter with $1.9-\mu \mathrm{m}$ resonator radii. (Color online only.) 
Table 2 Dependence of resonant wavelength and quality factor of the filter on microdisks resonator radii.

\begin{tabular}{ccc}
\hline \hline Resonator Radius $(\mu \mathrm{m})$ & $\lambda$ Resonant $(\mu \mathrm{m})$ & Quality Factor \\
\hline 1.80 & 1516.60 & 2500 \\
1.90 & 1520.31 & 5100 \\
2.00 & 1528.10 & 4900 \\
2.10 & 1530.10 & 7300 \\
\hline \hline
\end{tabular}

The fabricated structures were subsequently characterized using a micrometric optical setup for precise light coupling, a broadband near-IR (NIR) light source, and an optical spectrum analyzer. The use of surface grating couplers $^{11}$ as optical in- and output coupling devices to/ from the photonics structure sets the light polarization state in the bus waveguide to TE and limits the spectrum range to 1510 to $1590 \mathrm{~nm}$. The transmitted signals are normalized with respect to a straight reference waveguide with the same path length. Therefore, the wavelength-dependence of the in- and output losses of the grating couplers is removed.

The experimental transmitted and dropped spectra of the optical add-drop filter with resonator radii $R=1.9 \mu \mathrm{m}$ are shown in Fig. 6. In the wavelength range 1510 to $1590 \mathrm{~nm}$, two resonance peaks are present, at $1520.31 \mathrm{~nm}$ and $1579.93 \mathrm{~nm}$, bringing the FSR of the filter to $59.62 \mathrm{~nm}$. Both resonant peaks are actually a combination of two peaks of very nearby resonance wavelength originating from the slight difference in the optical circumference between the two microdisk resonators. Both peaks have high-

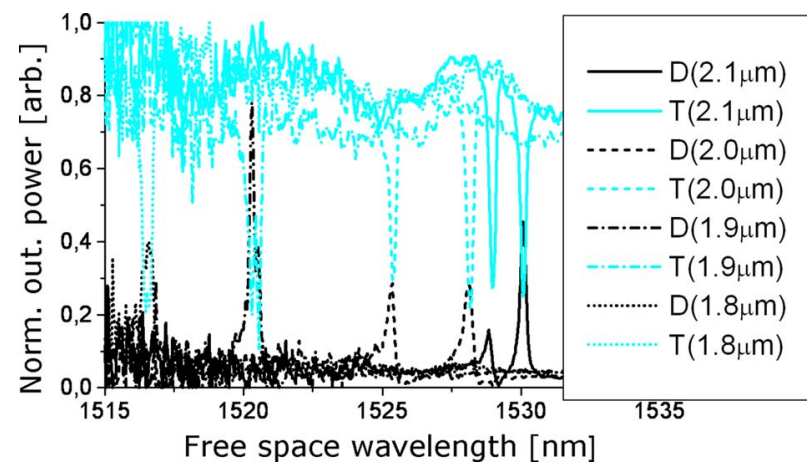

Fig. 7 Comparison of the resonant peak position for add-drop filters with resonators radii ranging between 1.8 and $2.1 \mu \mathrm{m}$.

quality factors. In the case of the peak at $1520.31 \mathrm{~nm}$, the quality factor is 5100 (if only the first sharp part of the peak is taken into account it reaches 11,700) while for the peak at $1579.93 \mathrm{~nm}$ the quality factor is 2700 . Similar measurements were performed for the add-drop filter structures with microdisk resonator radii ranging from 1.80 to $2.10 \mu \mathrm{m}$ (Fig. 7). All the results are compared in Table 2.

The resonant peaks of the filters with different radii are well separated. The resonant wavelength increment with increasing ring radius, observed in this wavelength range, is pure geometrical coincidence, as the peaks do not correspond to the same azimuth mode number (a variation of disk radius of $100 \mathrm{~nm}$ induces a shift in resonant wavelength of about $75 \mathrm{~nm}$, which is slightly larger than the FSR).

Table 3 Experimental parameters of the $4 \times 4$ optical network for all combinations of the input and output ports: resonant wavelength $\left(\lambda_{\mathrm{RES}}\right)$, resonant peak quality factor $(Q)$, ratio between dropping efficiency, and corresponding nonresonant transmission attenuation $(\Delta P)$.

\begin{tabular}{|c|c|c|c|c|c|}
\hline & & $\mathrm{T} 1$ & $\mathrm{~T} 2$ & T3 & T4 \\
\hline \multirow[t]{3}{*}{ M1 } & $\lambda_{\mathrm{RES}}$ & 1523.00 nm & $1527.96 \mathrm{~nm}$ & $1519.70 \mathrm{~nm}$ & Non resonant \\
\hline & $Q$ & 2600 & 2300 & 2700 & \\
\hline & $\Delta P$ & $>30 \mathrm{~dB}$ & $12 \mathrm{~dB}$ & $>30 \mathrm{~dB}$ & \\
\hline \multirow[t]{3}{*}{ M2 } & $\lambda_{\mathrm{RES}}$ & $1527.48 \mathrm{~nm}$ & $1530.76 \mathrm{~nm}$ & Non resonant & $1519.76 \mathrm{~nm}$ \\
\hline & $Q$ & 2250 & 2300 & & 3300 \\
\hline & $\Delta P$ & $10 \mathrm{~dB}$ & $12 \mathrm{~dB}$ & & $15 \mathrm{~dB}$ \\
\hline \multirow[t]{3}{*}{ M3 } & $\lambda_{\text {RES }}$ & 1520.48 nm & Non resonant & $1530.76 \mathrm{~nm}$ & 1528.08 nm \\
\hline & $Q$ & 2200 & & 2650 & 1600 \\
\hline & $\Delta P$ & $18 \mathrm{~dB}$ & & $10 \mathrm{~dB}$ & $20 \mathrm{~dB}$ \\
\hline \multirow[t]{3}{*}{ M4 } & $\lambda_{\mathrm{RES}}$ & Non resonant & $1520.48 \mathrm{~nm}$ & 1527.52 nm & $1523.00 \mathrm{~nm}$ \\
\hline & $Q$ & & 1450 & 2500 & 3200 \\
\hline & $\Delta P$ & & $>30 \mathrm{~dB}$ & $8 \mathrm{~dB}$ & $>30 \mathrm{~dB}$ \\
\hline
\end{tabular}




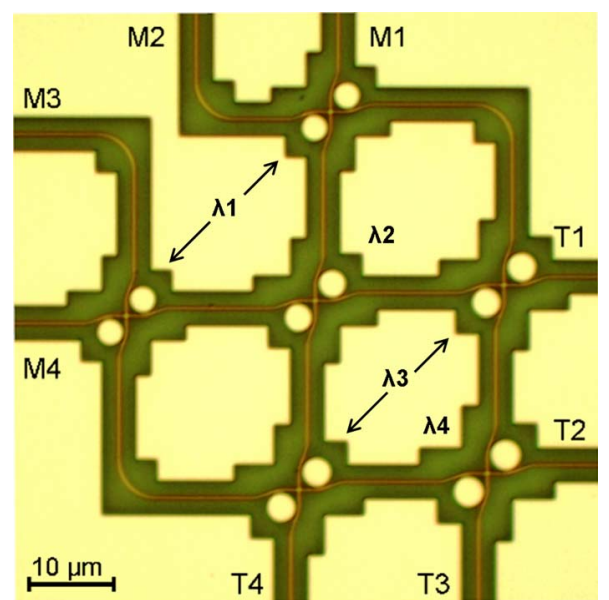

Fig. 8 Microscope photograph of the optical $4 \times 4$ network.

\section{Design and Validation of the $4 \times 4$ Optical Network}

A $4 \times 4$ network was fabricated at IMEC using the same technology and waveguide dimensions as for the aforementioned isolated filters. To enhance the resonant dropping efficiency, the coupling gaps were slightly reduced to $180 \mathrm{~nm}$. The network is composed of six filters grouped in four rows (shown on a diagonal in Fig. 8) with resonator radii of $1.8(\lambda 1), 1.9(\lambda 2), 2.0(\lambda 3)$, and $2.1 \mu \mathrm{m}(\lambda 4)$. The transmitted spectra at ports $\mathrm{T} 1$ to $\mathrm{T} 4$ for light injected at port M3 are shown in Fig. 9. The peaks correspond to the signals dropped to ports $\mathrm{T} 1, \mathrm{~T} 3$, and $\mathrm{T} 4$ under resonant condition, and the spectrum with the dips is the signal transmitted to port $\mathrm{T} 2$.

The relatively low output power level $(\approx-5 \mathrm{~dB})$ is most probably due to the influence of the nonoptimized waveguide intersection design causing moderate transmission loss and crosstalk. The resonant peaks have significantly different shape (closeup at the bottom of Fig. 9) and clearly emphasize the kind of problems that can be encountered when designing and fabricating optical networks based on this type of filters.

As expected, the signal dropped to port T1 (by router $\lambda 1, R=1.8 \mu \mathrm{m})$ shows one resonant peak at the center wavelength of $1520.48 \mathrm{~nm}$ with a quality factor of 2200 and an extinction ratio of $20 \mathrm{~dB}$. On the contrary, the signal dropped to port $\mathrm{T} 4$ (by router $\lambda 3, R=2.0 \mu \mathrm{m}$ ) shows a double peak at 1528.00 and $1528.68 \mathrm{~nm}$. This double peak originates from a slight difference in disk radii ( $\sim 0.9 \mathrm{~nm}$ ). As for the signal dropped to port T3 (by router $\lambda 4, R=2.1 \mu \mathrm{m})$, it also shows two separate resonant peaks (1530.76 and $1533.84 \mathrm{~nm})$, corresponding to a difference in disk radii of about $4.2 \mathrm{~nm}$. Despite these imperfections, the network still allows communication between all input and output ports. The quality factors of the resonant peaks are relatively high, ranging from 1450 to 3300 . The experimental parameters of the network for light injection to each input port are summarized in Table 3.
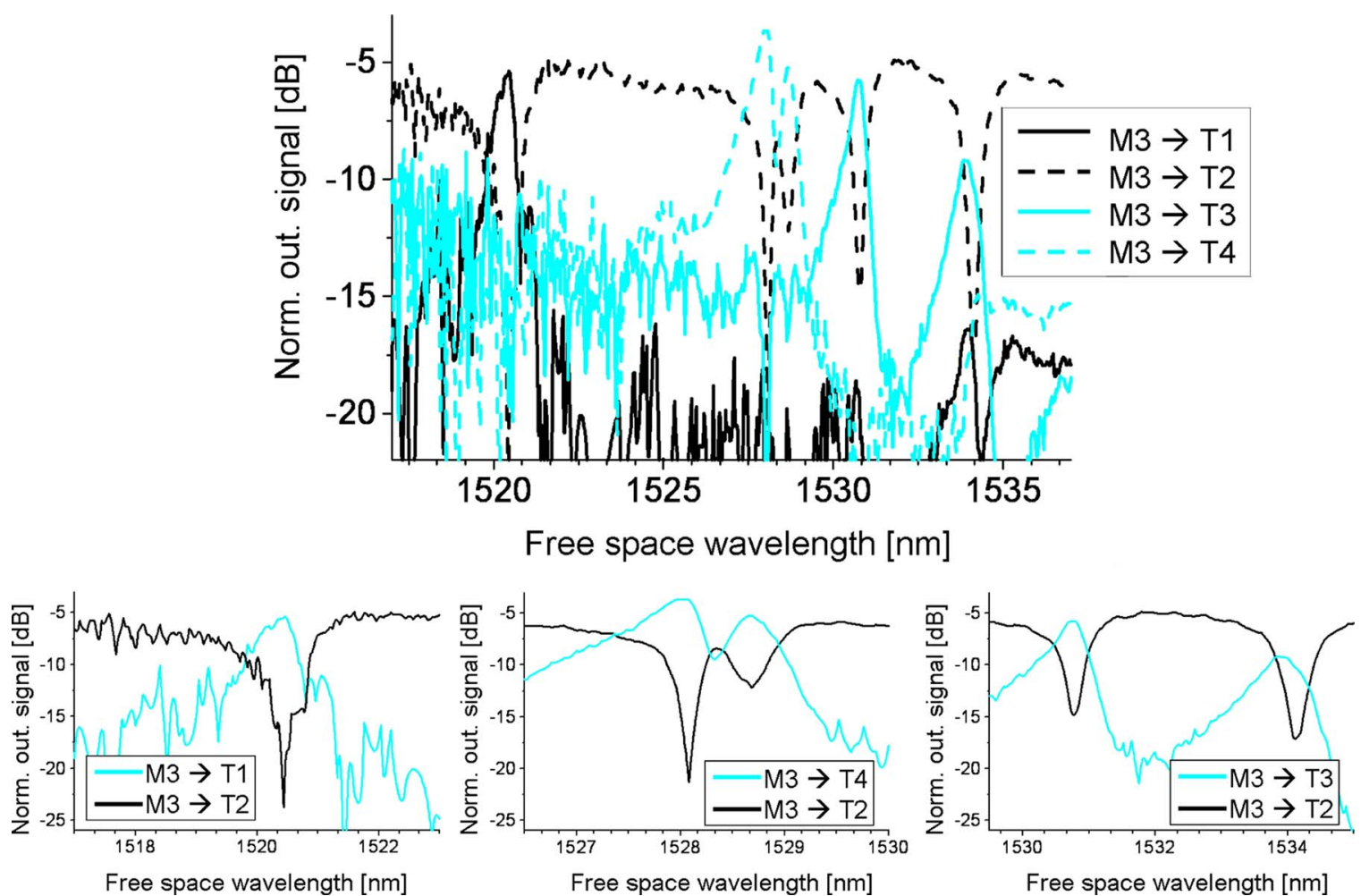

Fig. 9 Transmission spectra of the optical $4 \times 4$ network, signal injection is to port M3. 


\section{Conclusions}

We designed, fabricated, and validated an optical $4 \times 4$ network based on compact symmetric add-drop filters composed of a pair of microdisk resonators side-coupled to a waveguide intersection. Although the fabricated network was not optimized in terms of occupied chip area, it requires only a surface smaller than $50 \times 50 \mu \mathrm{m}^{2}$. It is the most compact device of this type demonstrated to date, providing complete $4 \times 4$ communication. ${ }^{12,13}$

The proper operation of the device was demonstrated, even though the quality of the transfer function could be improved. For instance, an active control of the resonant wavelengths (e.g., using integrated heaters) as well as more efficient intersections ${ }^{14}$ could be implemented, with the hope to demonstrate a fully functional device for on-chip optical communication.

\section{References}

1. B. E. Little, J. S. Foresi, G. Steinmeyer, E. R. Thoen, S. T. Chu, H. A Haus, E. P. Ippen, L. C. Kimerling, and W. Greene, "Ultra-compact $\mathrm{Si}-\mathrm{SiO}_{2}$ microring resonator optical channel dropping filters," IEEE Photonics Technol. Lett. 10, 549-551 (1998).

2. K. De Vos, I. Bartolozzi, E. Schacht, P. Bienstman, and R. Baets, "Silicon-on-insulator microring resonator for sensitive and label-free biosensing," Opt. Express 15, 7610-7615 (2007).

3. Q. Xu, S. Manipatruni, B. Schmidt, J. Shakya, and M. Lipson, "12.5 Gbit/s carrier-injection-based silicon microring silicon modulators," Opt. Express 15, 430-436 (2007).

4. P. Rojo Romeo, J. Van Campenhout, P. Regreny, A. Kazmierczak, C. Seassal, X. Letartre, G. Hollinger, D. Van Thourhout, R. Baets, J. M. Fedeli, and L. Di Cioccio, "Heterogeneous integration of electrically driven microdisk based laser sources for optical interconnects and photonic ICs," Opt. Express 14, 3864-3871 (2006).

5. P. Dumon, W. Bogaerts, V. Wiaux, J. Wouters, S. Beckx, J. Van Campenhout, D. Taillaert, B. Luyssaert, P. Bienstman, D. Van Thourhout, and R. Baets, "Low-loss SOI photonic wires and ring resonators fabricated with deep UV lithography," IEEE Photonics Technol. Lett. 16(5), 1328-1330 (2004).

6. A. Kazmierczak, M. Briere, E. Drouard, P. Rojo-Romeo, I O’Connor, X. Letartre, F. Gaffiot, L. El Melhaoui, P. Lyan, and J. M. Fedeli, "Design and characterisation of optical networks on chip," in Proc., 12th Eur. Conf. on Integrated Optics ECIO '05, Grenoble, France, pp. 188-191 (2005).

7. A. Kazmierczak, M. Brière, E. Drouard, P. Bontoux, P. Rojo-Romeo, I. O'Connor, X. Letartre, F. Gaffiot, R. Orobtchouk, and T. Benyattou, "Design, simulation and characterization of a passive optical add-drop filter in silicon-on-insulator technology," IEEE Photonics Technol. Lett. 17(7), 1447-1449 (2005).

8. E. J. Klein, D. H. Geuzebroek, H. Kelderman, G. Sengo, N. Baker, and A. Driessen, "Reconfigurable optical add-drop multiplexer using microring resonators," IEEE Photonics Technol. Lett. 17(11), 23582360 (2005)

9. I. O'Connor and F. Gaffiot, "On-chip optical interconnect for lowpower," in Ultra Low-Power Electronics and Design, E. Macii, Ed., Kluwer, Boston (2004)

10. W. Bogaerts, R. Baets, P. Dumon, V. Wiaux, S. Beckx, D. Taillaert, B. Luyssaert, J. Van Campenhout, P. Bienstman, and D. Van Thourhout, "Nanophotonic waveguides in silicon-on-insulator fabricated with CMOS technology," J. Lightwave Technol. 23(1), 401412 (2005).

11. D. Taillaert, F. Van Laere, M. Ayre, W. Bogaerts, D. Van Thourhout, P. Bienstman, and R. Baets, "Grating couplers for coupling between optical fibers and nanophotonic waveguides," Jpn. J. Appl. Phys. 45(8A), 6071-6077 (2006).

12. F. Xu and A. W. Poon, "Silicon cross-connect filters using microring resonator coupled multimode-interference-based waveguide crossings," Opt. Express 16, 8649-8657 (2008).
13. N. Sherwood-Droz, H. Wang, L. Chen, B. G. Lee, A. Biberman, K. Bergman, and M. Lipson, "Optical $4 \times 4$ hitless silicon router for optical networks-on-chip (NoC)," Opt. Express 16, 15915-15922 (2008)

14. W. Bogaerts, P. Dumon, D. Van Thourhout, and R. Baets, "Low-loss, low-crosstalk crossings for SOI nanophotonic waveguides," Opt. Lett. 32(19), 2801-2803 (2007).

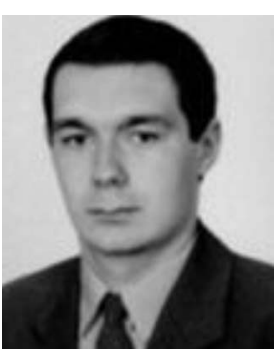

Andrzej Kaźmierczak graduated with honors from the Technical University of Łodź, Poland, in 2003. He conducted his PhD research at the T. U. Łodź, Poland, and at Ecole Centrale de Lyon, France, and received his PhD in 2007. He currently holds an R\&D engineer position at Multitel a.s.b.I. (Mons, Belgium), coordinating multitel activities in SABIO (Ultrahigh sensitivity SlotwAveguide BIOsensor on a highly integrated chip for simultaneous diagnosis of multiple diseases) and PHODYE (New PHOtonic Systems on a Chip based on DYEs for Sensor Applications scalable at Wafer Fabrication), European Union (EU)-founded projects. His scientific interests include integrated passive and active photonics devices and optical sensors. He has contributed to about 30 scientific communications in journals and at international conferences.

Fabian Dortu received his MSc degree in physical engineering from the Université de Liège, Belgium, in 2001 and his PhD degree in 2009 from the Katholieke Universiteit Leuven in collaboration with the Interuniversity Micro-Electronics Centre (IMEC), Leuven, on the development of optical methods for the characterization of ultrashallow junctions. He is currently with the Department of Applied Photonics at Multitel (Mons). His research interests include the simulation and modeling of nonlinear effects in light semiconductor interaction, planar photonics, and fiber Bragg gratings.

Domenico Giannone has led the Applied Photonics Department of Multitel since 2003. Before joining Multitel he was involved in several aspects of fiber Bragg grating technology and was part of Dr. Raman Kashyap's team at British Telecom Laboratories, United Kingdom. He studied for his PhD degree with the Photonics Research Group, Aston University, Birmingham, United Kingdom. He is author and coauthor of more than 20 journal and conference papers on fiber lasers and fiber Bragg grating technology.

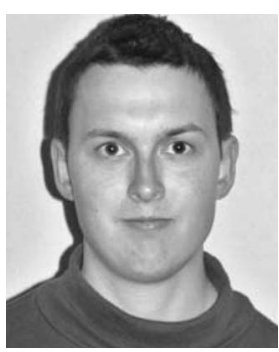

Wim Bogaerts completed his studies in engineering (applied physics) at Ghent University in 1998 and joined the departments of information technology (INTEC) at both Ghent University and the Interuniversity Microelectronics Center (IMEC), where he received his $\mathrm{PhD}$ degree in 2004. In the photonics research group, he specialized in the modeling, design, and fabrication of nanophotonic components. He is currently active as a postdoctoral fellow of the Flemish Research Foundation (FWO-Vlaanderen) and he coordinates the fabrication of nanophotonic components in SOI in IMEC, as a part of the European Network of Excellence ePIXnet. He is a member of IEEE-LEOS and OSA.

Emmanuel Drouard received his PhD degree from the University of Aix-Marseille III in 2003 on the design and experimental study of integrated optics devices made by $\mathrm{Ti}$ ion implantation in silica. He joined LEOM (now INL) in 2003 and he is now associate professor at the Ecole Centrale de Lyon. His research interests are the modeling and simulation of integrated optics refractive and diffractive devices for optical interconnects. 


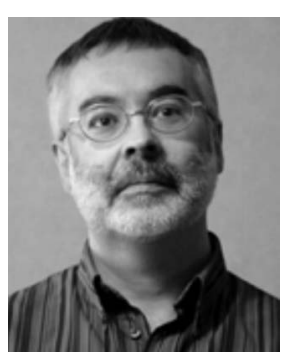

Pedro Rojo-Romeo received his InG Dipl degree in physics in 1981 from the Institut National des Sciences Appliquées (INSA) in Lyon, France, and his PhD degree in electronic devices in 1984 from INSA (Lyon). Since 1988 he has been an associate professor (since 1988) in electronics at Ecole Centrale de Lyon, France, at the Institute of Nanotechnologies of Lyon (INL). His primary research interests include electrical and optical devices fabrication technology, characterization of microelectronic and optoelectronic micronanodevices. He is also involved in optical interconnection systems, nanotechnologies, and optical integrated circuits technologies. His recent research areas are heterogeneous integration of III to V structures on Si-based substrates, the development of CMOS-compatible III to $\mathrm{V}$ nanolasers coupled to $\mathrm{Si}$ waveguides (programs FP6-STREPPICMOS, FP7-STREP-WADIMOS, French program RMNT HETEROPT), the development of novel 2.5-D photonic crystal lasers for guided and free-space laser emission (French program PNANO HEVICAL, FP7-PI-HELIOS), the localization of nanostructures on a patterned surface using e-beam lithography techniques, the localization of InAs quantum dots on InP for single-photon sources (French program NANOQUB), and the localized growth of III to V nanowires on $\mathrm{Si}$.

Frederic Gafflot: biography and photograph not available. 